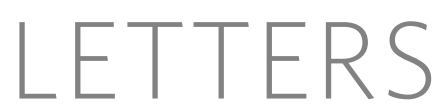

\title{
Self-assembly of a nanoscale DNA box with a controllable lid
}

\author{
Ebbe S. Andersen ${ }^{1,2,3}$, Mingdong Dong ${ }^{1,2,4} \uparrow$, Morten M. Nielsen ${ }^{1,2,3}$, Kasper Jahn ${ }^{1,2,3}$, Ramesh Subramani ${ }^{1,2,4}$, \\ Wael Mamdouh ${ }^{1,2,4}$, Monika M. Golas ${ }^{5,8}$, Bjoern Sander ${ }^{6,8}$, Holger Stark ${ }^{8,9}$, Cristiano L. P. Oliveira ${ }^{2,7}$, \\ Jan Skov Pedersen ${ }^{2,7}$, Victoria Birkedal ${ }^{2}$, Flemming Besenbacher ${ }^{1,2,4}$, Kurt V. Gothelf ${ }^{1,2,7}$ \& Jørgen Kjems ${ }^{1,2,3}$
}

The unique structural motifs and self-recognition properties of DNA can be exploited to generate self-assembling DNA nanostructures of specific shapes using a 'bottom-up' approach ${ }^{1}$. Several assembly strategies have been developed for building complex threedimensional (3D) DNA nanostructures ${ }^{2-8}$. Recently, the DNA 'origami' method was used to build two-dimensional addressable DNA structures of arbitrary shape ${ }^{9}$ that can be used as platforms to arrange nanomaterials with high precision and specificity ${ }^{9-13}$. A longterm goal of this field has been to construct fully addressable 3D DNA nanostructures ${ }^{14,15}$. Here we extend the DNA origami method into three dimensions by creating an addressable DNA box $42 \times 36 \times 36 \mathrm{~nm}^{3}$ in size that can be opened in the presence of externally supplied DNA 'keys'. We thoroughly characterize the structure of this DNA box using cryogenic transmission electron microscopy, small-angle X-ray scattering and atomic force microscopy, and use fluorescence resonance energy transfer to optically monitor the opening of the lid. Controlled access to the interior compartment of this DNA nanocontainer could yield several interesting applications, for example as a logic sensor for multiplesequence signals or for the controlled release of nanocargos.

We designed the DNA box by using a recently developed software package $^{16}$ to fold six DNA origami sheets along the circular, singlestranded DNA genome of the M13 bacteriophage (faces indicated with the letters A-F, Fig. 1a). The software built atomic models of the six interconnected sheets (Fig. 1b), which were subsequently arranged to form a 3D box (Fig. 1c). We then constructed the staple strands that fold the box by bridging the edges, resulting in a 'cuboid' structure of external size $42 \times 36 \times 36 \mathrm{~nm}^{3}$ (design details in Supplementary Notes 1-3). This particular design was chosen for several reasons: to use the entire M13 sequence, to ensure a circular folding path through the faces and to introduce faces that have the characteristics of lids with 'hinges' composed of scaffold linkers. Finally, the lid was functionalized with a lock-key system to control its opening (Supplementary Fig. 1).

The designed DNA structure formed by self-assembly after we heat annealed the 220 staple strands onto the single-stranded M13 DNA, resulting in highly homogenous structures migrating as one distinct band in native gel electrophoresis (Supplementary Fig. 2). Initially, a sample without the edge-linking staple strands was imaged in liquid using atomic force microscopy (AFM), revealing that the six DNA sheets formed efficiently and, in most instances, aligned in two parallel rows (Fig. 2a), which is compatible with helical stacking interactions between the edges of the DNA sheets ${ }^{9}$ (Supplementary Fig. 3a).

In a second assembly reaction, we used 59 staple strands, connecting the edges, to form the box shape, and subsequent AFM imaging revealed box-like particle structures (Fig. 2b). Analysis of the highresolution AFM images of individual particles revealed $x$ and $y$ dimensions that were in good agreement with the shape and dimensions of the designed DNA box. However, the measured heights of these particles varied between 4 and $12 \mathrm{~nm}$ (Supplementary Fig. 3b),
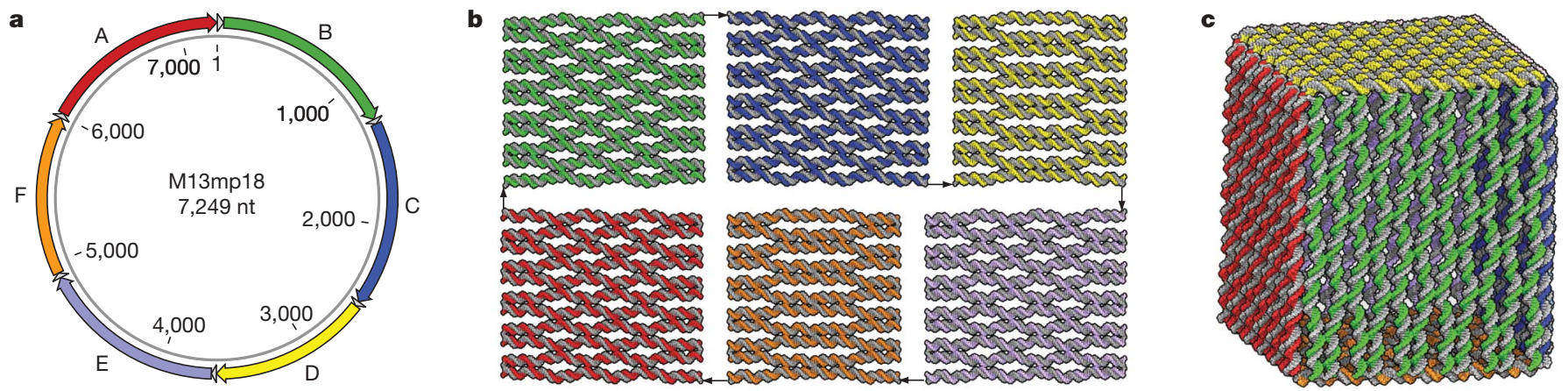

Figure 1 | Design of a DNA origami box. a, Sequence map of the circular, single-stranded DNA genome of the M13 bacteriophage with regions used to fold the six DNA sheets shown as coloured arrows (A-F). Base numbering starts from a 44-nucleotide spacer region between sheets $A$ and $B$ that contains a stable hairpin structure ${ }^{9}$. Spacers of 33 nucleotides are positioned between each face. $\mathbf{b}, \mathbf{c}$, Molecular models of the six DNA sheets in a flat and cubic higher-order structure, respectively. The six DNA sheets are colourcoded as in a.

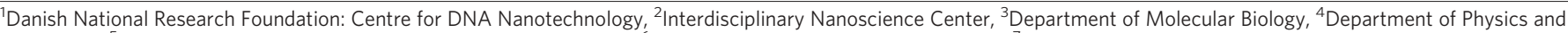

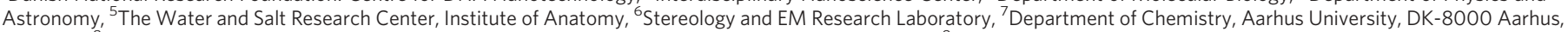

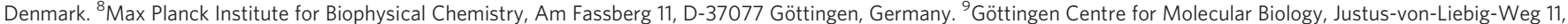

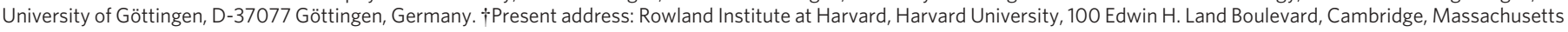
02142, USA. 
a

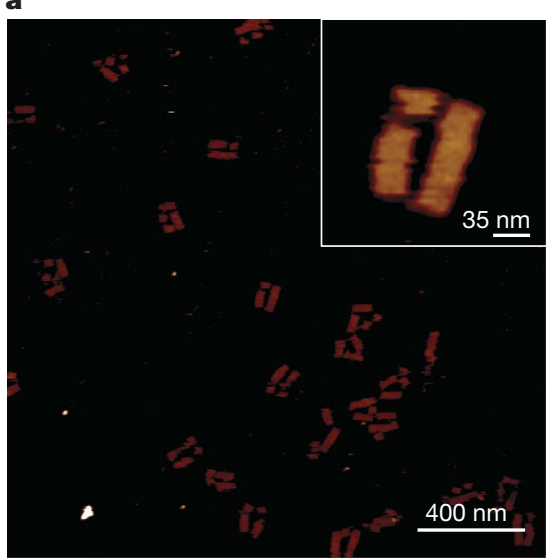

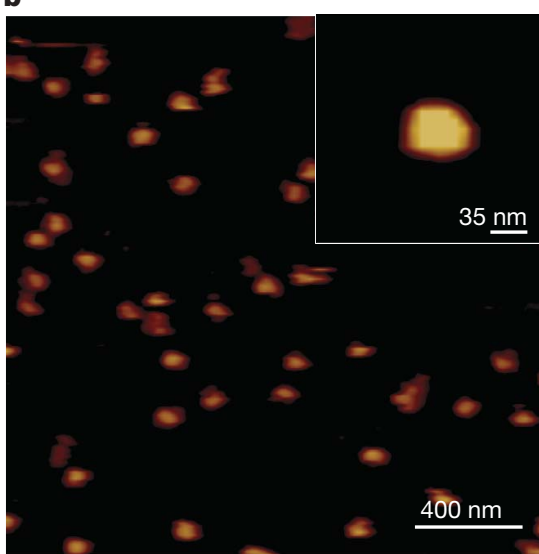

c

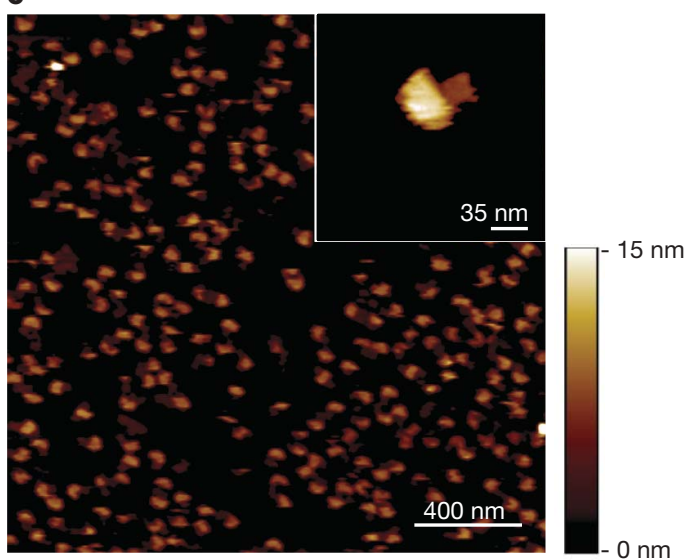

Figure 2 | AFM imaging of two- and three-dimensional DNA origami structures. a, AFM image of a sample in which the six DNA sheets were folded along the M13 backbone. Inset, magnified view of a preferred arrangement of the six sheets. b, AFM image of a sample in which the edges of the DNA sheets were linked to form a box. Inset, magnified view of a box-

which is most likely caused by distortion of the hollow, soft DNA box by the force exerted by the AFM tip. In support of this argument, we observed that prolonged scanning of individual 3D DNA structures results in their collapse into flat structures one or two DNA sheets in height (Supplementary Fig. 4a).

To further investigate the predictability of the 3D folding, we assembled an open DNA box in which the staple strands closing lid D were omitted. AFM images revealed flat particles two DNA sheets in height with a one-sheet extension of dimensions similar to lid D (Fig. 2c), which probably corresponds to a sideway collapse of the structure (Supplementary Fig. 4b). In conclusion, the AFM images obtained before and after addition of the staple strands connecting the edges of the DNA box strongly indicate the formation of box-shaped structures.

To gain stronger evidence for the formation of the DNA box, we performed cryogenic transmission electron microscopy (cryo-EM) on
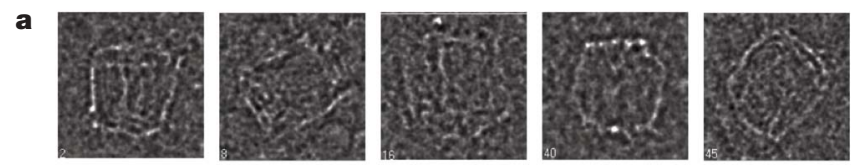

b

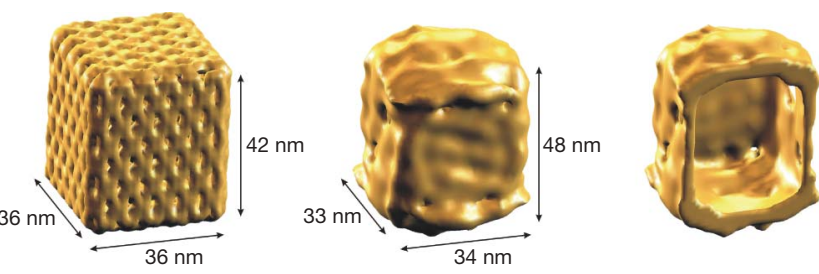

c

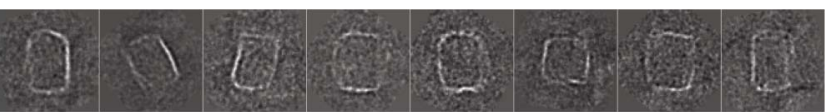

d

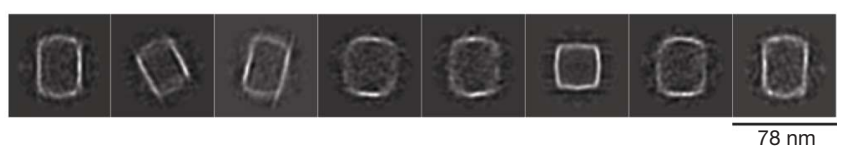

Figure 3 | Characterization of DNA origami box by cryo-EM and smallangle X-ray scattering (SAXS). a, Single-particle cryo-EM images of boxshaped assemblies. $\mathbf{b}$, Single-particle reconstruction of the DNA box applying D2 symmetry. Left, theoretical model. Middle, surface representation of the cryo-EM map. Right, cut-open view showing the interior cavity of the cryo-EM map. c, d, Comparison of the class averages of the DNA boxes (c) with the corresponding two-dimensional re-projections of the 3D cryo-EM map (d). e, Experimental SAXS data (circles) with like particle. c, AFM image of a sample in which one lid of the DNA box was left open. Inset, magnified view of a structure in which the lid is protruding from the body of the box. The colour scale shows the height above the surface in the range $0-15 \mathrm{~nm}$.

a sample of the assembly. Individual DNA boxes showed prominent edges (Fig. 3a) resulting from the increased density of the lateral faces oriented more or less parallel to the incident electron beam. Nearly all of the individual particles (90-95\%) seen in the cryo-EM images had an overall box-like shape. A total of 8,987 defocus-corrected box images were subjected to single-particle image processing, and class averages with $\sim 10-30$ members on average showed the main structural features of the DNA box (Fig. 3c). For 337 class averages, electrondense edges could be used to measure the short and long side lengths of the box, yielding dimensions of $44.6 \pm 3.7 \mathrm{~nm}$ by $36.8 \pm 3.2 \mathrm{~nm}$ (Supplementary Fig. 5). For 3D reconstruction, class averages were selected using the $3 \mathrm{D}$ error as selection criterion (Fig. 3b). The shape of the 3D cryo-EM map of the DNA box was consistent with the class averages and the corresponding two-dimensional re-projections (Fig. 3c, d). Overall, the 3D cryo-EM map revealed a hollow box-like structure with slightly convex bending of the small faces and slightly

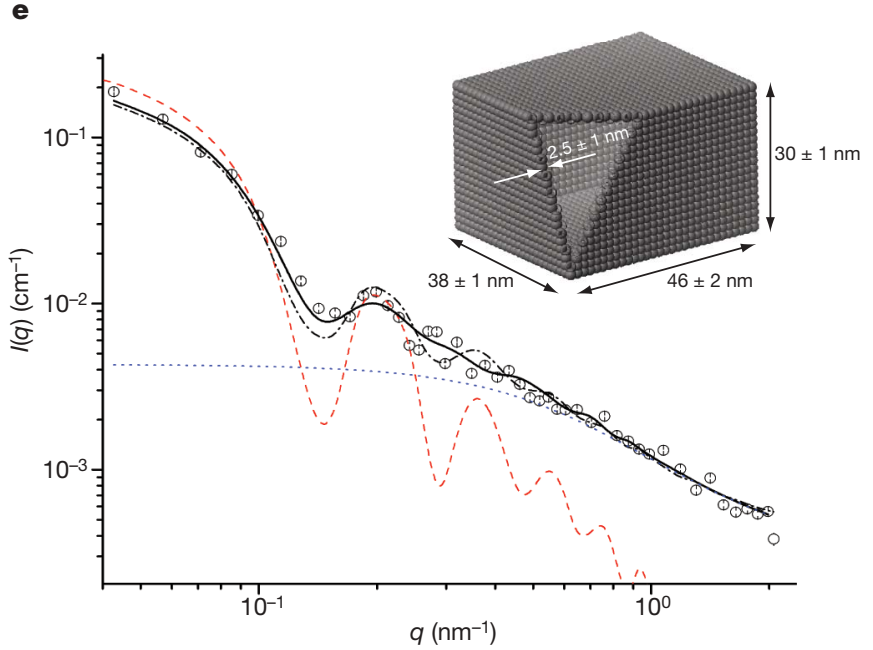

corresponding fits from different approaches: red dashed curve, fit using the theoretical atomic coordinates for the box; blue dotted curve, typical Debye background ${ }^{25}$ for modelling the excess oligonucleotides; dash-dot curve, fit using the theoretical atomic coordinates for the box with the Debye background added; solid curve, fit using a semi-analytical model for a box with three different side lengths with the Debye background added. $I(q)$, SAXS intensity; $q$, momentum transfer modulus (Methods). Inset, semianalytical box model with the estimated side lengths and wall thickness. 
concave bending of the large faces (Fig. 3b; see Supplementary Fig. 6 for additional views of the $3 \mathrm{D}$ map). We suggest that the convex and concave bending of the faces may reflect the difference in design of the small and large faces. The dimensions of the $3 \mathrm{D}$ reconstruction are compatible with $82 \%$ of the measured class averages, whereas the theoretical model is compatible with $61 \%$ of them (Supplementary Fig. 5). We conclude that the main fraction of the self-assembled structures had a hollow box-like shape that is very similar to the intended 3D design.

To analyse the native DNA origami box in solution and without a potentially disruptive sample fixation, we used dynamic light scattering and SAXS, which probe a large ensemble and can therefore determine whether or not the box is the major product of the self-assembly reaction. Analysis by dynamic light scattering showed there to be a contribution with a hydrodynamic radius $R_{\mathrm{h}}=24 \pm 4 \mathrm{~nm}$, which matches the calculated value of $25.2 \mathrm{~nm}$ from the atomic model, and a contribution with $R_{\mathrm{h}}=2.1 \mathrm{~nm}$, corresponding to nonannealed DNA oligonucleotides (Supplementary Fig. 7). The SAXS analysis yielded a characteristic profile of a well-defined particle and Fourier transformation analysis gave a single-particle self-correlation function characteristic of a hollow structure (data not shown). The scattering data was in good agreement with the atomic model of the DNA box structure (Fig. 3e). We also compared the experimental data with a theoretical model of a box with dimensions $2 a, 2 b$ and $2 c$ and wall thickness $t$ (Methods and Supplementary Fig. 8). This model can satisfactorily describe the scattering data and gave overall dimensions of $46 \pm 2 \mathrm{~nm}$ by $38 \pm 1 \mathrm{~nm}$ by $30 \pm 1 \mathrm{~nm}$ and a wall thickness of $2.5 \pm 1 \mathrm{~nm}$, which is close to the expected size and is consistent with the dimensions observed using cryo-EM. In conclusion, the SAXS data showed that the scattering derives mainly from box-shaped structures, providing further evidence of the successful self-assembly of 3D hollow boxes of well-defined dimensions in solution.

Previous studies have shown that DNA nanostructures can be dynamically manipulated by external DNA sequence signals $s^{7,17,18}$. Here we functionalized lid D of the DNA box with a dual lock-key system composed of DNA duplexes with sticky-end extensions to provide a 'toehold'19 for the displacement by externally added 'key' oligonucleotides (Supplementary Fig. 1). To detect the opening process of the DNA box lid, we inserted two fluorescent dyes, Cy3 and Cy5, into faces B and D, respectively (Fig. 4a and Supplementary Note 4). Efficient fluorescence resonance energy transfer (FRET) between the two fluorophores corresponds to a closed state in which the dyes are in close proximity (Fig. $4 \mathrm{~b}$, left). The addition of key oligonucleotides results in the opening of the lid, resulting in a decrease in the FRET efficiency as the distance between the two dyes increases (Fig. $4 b$, right).

The functionalized DNA box was assembled, purified and subjected to ensemble FRET measurements. We first studied the six faces without edge links and found emission only from primary excited Cy3, and only a dilution effect was observed upon addition of key oligonucleotides (Fig. 4c). By contrast, emission from the closed box sample had a Cy5 fluorescence peak at a wavelength of $665 \mathrm{~nm}$ that is consistent with a FRET signal with an estimated efficiency of $\sim 0.22$ (Fig. 4d). The addition of both keys led to a decreased Cy5 signal and an increased Cy3 signal (Fig. 4d), and the FRET efficiency decreased by $90 \%$, to $\sim 0.02$. The difference in response of the Cy5 and Cy3 signals may be caused by the difference in local environment of the two fluorophores. Cy5 was positioned on the side of a DNA helix, whereas Cy3 was positioned at the end of a DNA helix, where basestacking interactions ${ }^{20}$ may partly quench the fluorophore.

We measured the kinetics of the opening process (Fig. 4e) and found the FRET signal to decrease biexponentially, with an initial (fast) decay time of $\sim 40$ s upon key addition. The effect was specific, as no significant reduction was observed upon addition of an unrelated oligonucleotide (Fig. 4e). Order-of-addition experiments showed that both keys are required for full decrease of the FRET signal (Supplementary Fig. 9). This indicates that a closed box can be programmed to open in response to at least two external signals (representing an AND gate). The box lid could potentially be designed to close again in the presence of specific signals (representing a NOT gate), and because the DNA box
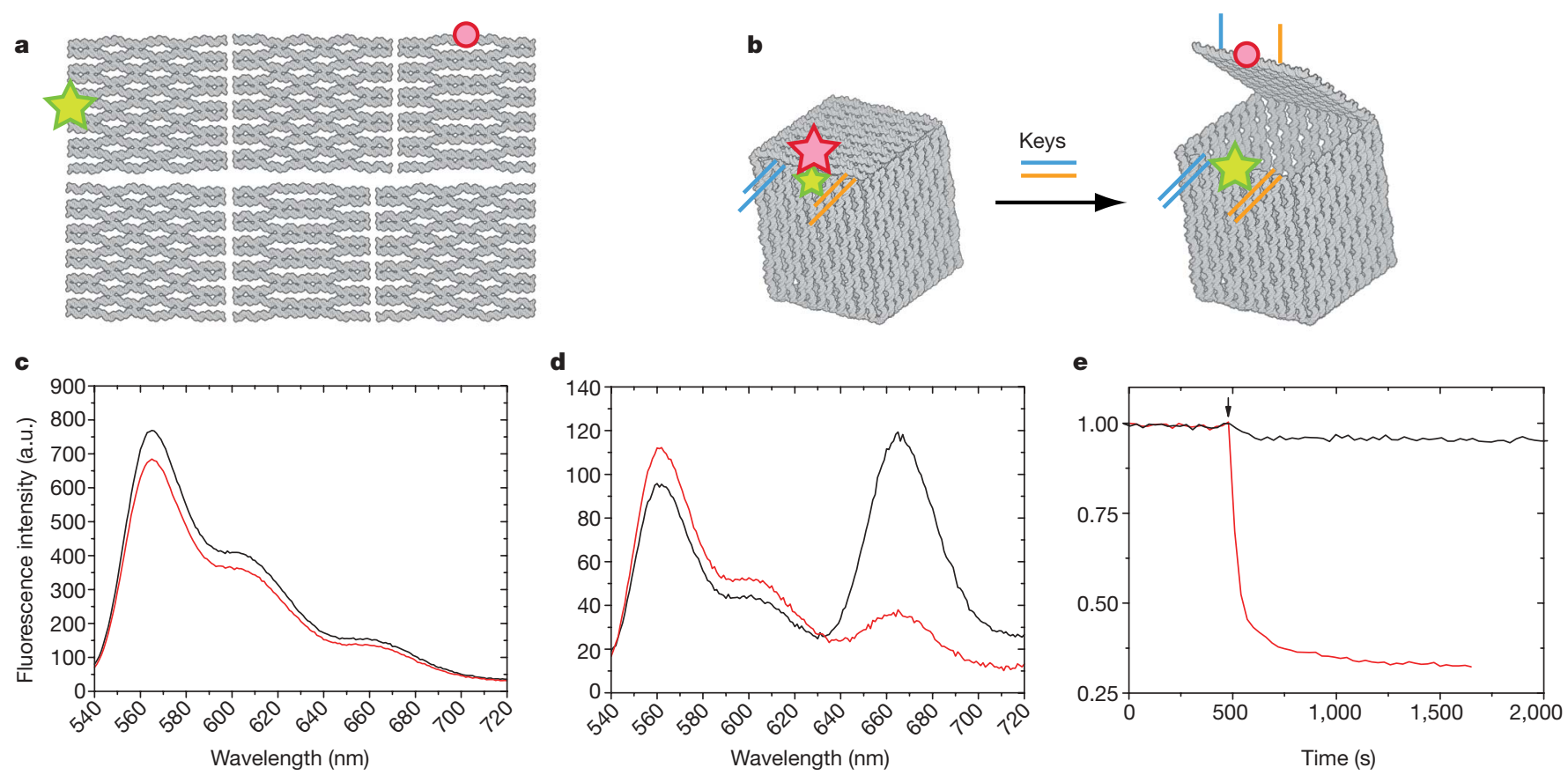

Figure 4 | Programmed opening of the box lid. a, b, Illustrations of the unlinked faces of the box (a) and the controlled opening of the box lid (b). The emission from the $\mathrm{Cy} 5$ and $\mathrm{Cy} 3$ fluorophores are marked with red and green stars, respectively. Loss of emission from Cy5 is denoted by a red circle and the independent lock-key systems are indicated in blue and orange. c, Ensemble FRET measurements of the unlinked faces before (black curve) and 12 min (red curve) after the addition of keys. d, Ensemble FRET measurements of the closed box before (black curve) and 35 min after (red curve) the addition of keys. e, Kinetic study of change in emission of Cy5. Black arrow, time of addition of key oligonucleotides (red curve) or an unrelated oligonucleotide (black curve). Initial fluorescence was normalized to one. a.u., arbitrary units. 
has two similar lids, D and F, they may be programmed individually (representing an OR gate). Thus, the lids of the DNA box have the potential to be uniquely programmed to respond to complex combinations of oligonucleotide sequences, for example cellular messenger RNAs or micro RNAs.

We have designed, synthesized and thoroughly characterized a megadalton-sized DNA box with a cavity that is large enough to contain, for example, a ribosome or a poliovirus. Furthermore, we have demonstrated dynamic control and programmability of the box lid. In an earlier study, we demonstrated that DNA origami structures can be designed that have tunable flexible properties ${ }^{16}$, and here we further show that dynamic changes can be induced by sensing external signals in the environment. The application of such a 'nanorobotic' device could be to restrict the transport of material in or out of the box in a controlled fashion. Importantly, the opening mechanism of the DNA box operates under native conditions, which allows biologically active components like enzymes to be packaged to provide control of access to their relevant substrates. We note that the DNA box presented here has the potential to both sense and act, for example by combining a diagnostic sensor of complex signals with the controlled release of, or access to, a payload.

\section{METHODS SUMMARY}

Software. The software package used for the box design consists of a sequence editor and an extendable algorithm toolbox ${ }^{16}$. We developed a program for creating realistic 3D models, which facilitated the design of the 3D edge-to-edge staple strand crossovers. The software package is distributed as free software under the GNU General Public License version 3 (GPLv3) and documentation and tutorials are available at www.cdna.dk/origami. A specific tutorial for designing the DNA box is available (see Nature Protocols doi:10.1038/nprot.2009.75).

AFM. We deposited the samples on a mica surface and performed the AFM imaging in a buffer solution. The best AFM images of the DNA box were obtained when applying minimal loading forces and using optimized feedback parameters.

Cryo-EM. The sample was adsorbed on carbon film and plunge-frozen in liquid ethane. We made low-dose images under cryogenic conditions using a $4 k \times 4 k$ charge-coupled-device detector at $0.245 \mathrm{~nm}$ per pixel (ref. 21), and a total of 8,987 single-particle images were selected manually and analysed in the context of IMAGIC-5 (ref. 22).

SAXS. We collected data using a high-resolution set-up of a laboratory-based SAXS instrument ${ }^{23}$. The scattering data was analysed on the basis of a theoretical model for a rectangular box using home-written software, and with an atomiccoordinate-based model applying the program CRYSOL ${ }^{24}$.

FRET. To detect the opening process, we functionalized the box with $\mathrm{Cy} 3$ and Cy5 fluorophores and a lock-key system. Purified samples were investigated using ensemble FRET spectroscopy measurements. The opening of the box was directly monitored in fluorescence kinetic experiments, where DNA key oligonucleotides were added to the closed-box sample.

Full Methods and any associated references are available in the online version of the paper at www.nature.com/nature.

\section{Received 9 November 2008; accepted 6 March 2009.}

1. Seeman, N. C. An overview of structural DNA nanotechnology. Mol. Biotechnol. 37, 246-257 (2007).

2. Chen, J. H. \& Seeman, N. C. Synthesis from DNA of a molecule with the connectivity of a cube. Nature 350, 631-633 (1991).

3. Shih, W. M., Quispe, J. D. \& Joyce, G. F. A 1.7-kilobase single-stranded DNA that folds into a nanoscale octahedron. Nature 427, 618-621 (2004).
4. Goodman, R. P. et al. Rapid chiral assembly of rigid DNA building blocks for molecular nanofabrication. Science 310, 1661-1665 (2005).

5. Douglas, S. M., Chou, J. J. \& Shih, W. M. DNA-nanotube-induced alignment of membrane proteins for NMR structure determination. Proc. Natl Acad. Sci. USA 104, 6644-6648 (2007).

6. Andersen, F. F. et al. Assembly and structural analysis of a covalently closed nanoscale DNA cage. Nucleic Acids Res. 36, 1113-1119 (2008).

7. Yang, H. \& Sleiman, H. F. Templated synthesis of highly stable, electroactive, and dynamic metal-DNA branched junctions. Angew. Chem. Int. Ed. 47, 2443-2446 (2008).

8. He, Y. et al. Hierarchical self-assembly of DNA into symmetric supramolecular polyhedra. Nature 452, 198-201 (2008).

9. Rothemund, P. W. Folding DNA to create nanoscale shapes and patterns. Nature 440, 297-302 (2006).

10. Williams, B. A., Lund, K., Liu, Y., Yan, H. \& Chaput, J. C. Self-assembled peptide nanoarrays: an approach to studying protein-protein interactions. Angew. Chem Int. Ed. 46, 3051-3054 (2007).

11. Ke, Y., Lindsay, S., Chang, Y., Liu, Y. \& Yan, H. Self-assembled water-soluble nucleic acid probe tiles for label-free RNA hybridization assays. Science 319, 180-183 (2008).

12. Rinker, S., Ke, Y., Liu, Y., Chhabra, R. \& Yan, H. Self-assembled DNA nanostructures for distance-dependent multivalent ligand-protein binding. Nature Nanotechnol. 3, 418-422 (2008).

13. Sharma, J. et al. Toward reliable gold nanoparticle patterning on self-assembled DNA nanoscaffold. J. Am. Chem. Soc. 130, 7820-7821 (2008).

14. Seeman, N. C. Nucleic acid junctions and lattices. J. Theor. Biol. 99, 237-247 (1982).

15. Simmel, F. C. Three-dimensional nanoconstruction with DNA. Angew. Chem. Int. Ed. 47, 5884-5887 (2008).

16. Andersen, E. S. et al. DNA origami design of dolphin-shaped structures with flexible tails. ACS Nano 2, 1213-1218 (2008).

17. Ding, B. \& Seeman, N. C. Operation of a DNA robot arm inserted into a 2D DNA crystalline substrate. Science 314, 1583-1585 (2006).

18. Goodman, R. P. et al. Reconfigurable, braced, three-dimensional DNA nanostructures. Nature Nanotechnol. 3, 93-96 (2008).

19. Yurke, B., Turberfield, A. J., Mills, A. P., Simmel, F. C. \& Neumann, J. L. A DNAfuelled molecular machine made of DNA. Nature 406, 605-608 (2000).

20. Iqbal, A. et al. Orientation dependence in fluorescent energy transfer between Cy 3 and Cy 5 terminally attached to double-stranded nucleic acids. Proc. Natl Acad. Sci. USA 105, 11176-11181 (2008).

21. Sander, B., Golas, M. M. \& Stark, H. Advantages of CCD detectors for de novo three-dimensional structure determination in single-particle electron microscopy. J. Struct. Biol. 151, 92-105 (2005).

22. van Heel, M., Harauz, G., Orlova, E. V., Schmidt, R. \& Schatz, M. A new generation of the IMAGIC image processing system. J. Struct. Biol. 116, 17-24 (1996).

23. Pedersen, J. S. A flux- and background-optimized version of the NanoSTAR smallangle X-ray scattering camera for solution scattering. J. Appl. Crystallogr. 37, 369-380 (2004).

24. Svergun, D., Barberato, C. \& Koch, M. H. J. CRYSOL - A program to evaluate X-ray solution scattering of biological macromolecules from atomic coordinates. J. Appl. Crystallogr. 28, 768-773 (1995).

25. Debye, P. Molecular-weight determination by light scattering. J. Phys. Colloid Chem. 51, 18-32 (1947).

Supplementary Information is linked to the online version of the paper at www.nature.com/nature.

Acknowledgements We thank R. Rosendahl Hansen and J. Kristensen for technical assistance. This work was supported by grants from the Danish National Research Foundation to the Centre for DNA Nanotechnology and the Danish Research Agency through support to the Interdisciplinary Nanoscience Center, by the Federal Ministry of Education and Research, Germany (0311899), and by the Sixth Framework Program of the European Union through the Integrated Project '3D Repertoire' (H.S.).

Author Information The 3D map has been deposited in the EM Data Bank under the accession code EMD-1612. Reprints and permissions information is available at www.nature.com/reprints. Correspondence and requests for materials should be addressed to K.V.G. (kvg@chem.au.dk) or J.K. (jk@mb.au.dk). 


\section{METHODS}

Sample preparation. We prepared the m13mp18 DNA as described previously $^{16}$. The assembly reactions were performed in Tris-acetate-EDTA buffer with $12.5 \mathrm{mM} \mathrm{MgAc}$ (TAEM), $1.6 \mathrm{nM} \mathrm{M13}$ and fivefold excess of each oligonucleotide. The samples were heated to $95^{\circ} \mathrm{C}$ and cooled to $20^{\circ} \mathrm{C}$ in steps of $0.1{ }^{\circ} \mathrm{C}$ every $6 \mathrm{~s}$.

AFM. The sample ( $5 \mu \mathrm{l})$ was deposited on a freshly cleaved mica surface (Ted Pella) and left to adsorb for $5 \mathrm{~min}$. Then $200 \mu \mathrm{l}$ TAEM buffer was added to the liquid cell and the sample was scanned in tapping-mode using either an Agilent AFM series 5500 (Agilent Technologies) or a MultiMode SPM with a Nanoscope IV controller (Veeco Instruments) under ambient conditions. We used oxidesharpened triangular cantilevers (NP-S, Veeco Instruments) with a spring constant of $0.4 \mathrm{~N} \mathrm{~m}^{-1}$ and a normal tip radius of $10 \mathrm{~nm}$. After engagement, the tapping-amplitude set point was typically $0.5 \mathrm{~V}$ and the scan rates ranged from 1 to $2 \mathrm{~Hz}$. During the liquid AFM imaging, the highest resolution was in most cases obtained with minimal loading forces applied and using optimized feedback parameters. We obtained several AFM images, all $512 \times 512$ pixels, from separate locations across the mica surfaces to ensure reproducibility of the results. All the AFM images were analysed using Scanning Probe Image Processor (SPIP) software (Image Metrology ApS, version 4.7.4).

Sample preparation for cryo-EM. For cryo-EM, we supplemented the DNA box sample with $1 \mathrm{mM}$ spermidine. For adsorption of the particles, a thin carbon film was used that was prepared by evaporating carbon on a piece of freshly cleaved mica. About $25 \mu \mathrm{l}$ of the sample were filled into a well in an ice-cooled block, and particles were adsorbed on a carbon film for between $30 \mathrm{~min}$ and $12 \mathrm{~h}$. Subsequently, the floating carbon film was picked out from the particle solution by placing a copper EM grid covered with a perforated carbon film on top of the floating carbon film. After adding $5 \mu \mathrm{l}$ of the particle solution on one side of the EM grid, we mounted the grid in a home-made freeze-plunging device, blotted and immediately plunge-froze it in liquid ethane. The EM grids were stored in liquid nitrogen until image acquisition.

Electron cryomicroscopy. Specimen grids were transferred to the electron cryomicroscope (CM200 FEG electron microscope, Philips/FEI) under cryogenic conditions using a cryoholder (Gatan). The electron microscope was operated at an acceleration voltage of $160 \mathrm{kV}$ in low-dose mode. Images were taken on a $4 k \times 4 k$ CCD (charge-coupled-device) camera (TemCam-F415, TVIPS) with twofold binning of the pixels and $\times 122,000$ magnification (ref. 21). A total of 4,160 CCD camera exposures were collected.

Image processing. Image processing was performed in the framework of the IMAGIC-5 software package ${ }^{22}$. We manually selected 8,987 single-particle images of the DNA boxes, and corrected them for the contrast transfer function as described previously ${ }^{26}$. For image processing, the images were coarsened to a pixel size of $4.9 \AA$ at the specimen level. Images were subjected to three iterative rounds of multireference alignment using resampling to polar coordinates ${ }^{27}$ and multivariate statistical analysis. Aligned images were classified using hierarchical ascendant classification with moving-element refinement, resulting in class averages with $\sim 10-30$ class members on average. The $3 \mathrm{D}$ reconstruction was iteratively refined in three rounds of projection matching (pixel size, $9.8 \AA$ ), starting with 337 class averages that showed well-defined structural features. The $3 \mathrm{D}$ error was used to select the best class averages for $3 \mathrm{D}$ calculation. Thereby, the number of selected class averages was reduced to 250 and finally to 36 .

Determination of dimensions. For a graphical and statistical description of the size distribution of the DNA boxes (given as average plus/minus standard deviation), the long and short side lengths were measured for 337 experimental class averages that showed well-defined edges. These values were colour-coded according to their abundance and plotted graphically. The theoretical box model and the experimental 3D structure were re-projected into 1,654 two-dimensional, equally spaced re-projections that were measured to define the respective size distributions. Dynamic light scattering. The experiments were performed at a scattering angle of $90^{\circ}$ on an ALV instrument with a CGS-8F goniometer system equipped with an ALV-6010/EPP multi-tau digital correlator and a helium-neon diode laser (JDS Uniphase). ALV software was used to derive the hydrodynamic radius using cumulant analysis and inverse Laplace transforms. The samples were the same as used in the SAXS experiments, but measured at $23^{\circ} \mathrm{C}$.

SAXS. The experiments were performed in the laboratory-based SAXS instrument at the Department of Chemistry, Aarhus University ${ }^{23}$. The SAXS intensity was displayed as a function of the momentum transfer modulus $q(q=4 \pi \sin (\theta) /$ $\lambda$, where $\lambda$ is the radiation wavelength and $2 \theta$ is the scattering angle). Pure water was used as primary standard for absolute scale normalization. The sample concentration was very low $\left(\sim 0.1 \mathrm{mg} \mathrm{ml}^{-1}\right)$ and the data was collected in the high-resolution set-up covering $0.004 \AA^{-1}<q<0.21 \AA^{-1}$. The samples were injected into carefully rinsed quartz capillaries and placed in a thermostated block $\left(4^{\circ} \mathrm{C}\right)$ in the vacuum chamber of the instrument.

The modelling of the scattering data was performed using the expression

$$
I(q)=S_{\mathrm{C}_{1}} P_{\mathrm{BOX}}(q)+S_{\mathrm{C}_{2}} P_{\mathrm{DEB}}(q)+\text { Back }
$$

where $S_{\mathrm{C}_{i}}$ are the scale factors for the scattering components and Back is a constant background that might correct imperfect data reduction or the contribution from electron density fluctuations in the sample. $P_{\mathrm{BOX}}(q)$ is the scattering intensity of a rectangular box with sides $2 a, 2 b$ and $2 c$ and wall thickness $t$, given by

$$
P_{\mathrm{BOX}}(q)=\frac{2}{\pi} \int_{0}^{\pi / 2} \int_{0}^{\pi / 2}\left(A_{\mathrm{REC}}(q, a, b, c)-A_{\mathrm{REC}}(q, a-t, b-t, c-t)\right)^{2} \sin \alpha \mathrm{d} \alpha \mathrm{d} \beta
$$

where $\alpha$ and $\beta$ are polar angles of integration and $A_{\mathrm{REC}}(q, a, b, c)$ is the scattering amplitude of a rectangular box with sides $2 a, 2 b$ and $2 c$ (Supplementary Fig. 8), given by

$$
A_{\mathrm{REC}}=8 a b c \frac{\sin (q a \sin \alpha \sin \beta)}{q a \sin \alpha \sin \beta} \frac{\sin (q b \sin \alpha \cos \beta)}{q b \sin \alpha \cos \beta} \frac{\sin (q c \cos \alpha)}{q c \cos \alpha}
$$

$P_{\mathrm{DEB}}(q)$ is the scattering intensity of a Gaussian chain with a radius of gyration $R_{\mathrm{g}}$ (ref. 25):

$$
P_{\mathrm{DEB}}(q)=\frac{2\left(\exp \left(-q^{2} R_{\mathrm{g}}^{2}\right)-1+q^{2} R_{\mathrm{g}}^{2}\right)}{\left(q^{2} R_{\mathrm{g}}^{2}\right)^{2}}
$$

Because the sample had free oligonucleotides in the solution (also detected by dynamic light scattering measurements), it was necessary to add this intensity to take into account their contribution to the scattering.

The measured intensity is smeared by instrumental effects. To account for this, we use a resolution function ${ }^{28}$. The observed experimental intensity, $\tilde{I}(\langle q\rangle)$, is thus fitted by

$$
\tilde{I}(\langle q\rangle)=\int R(\langle q\rangle, q) I(q) \mathrm{d} q
$$

where $R(\langle q\rangle, q)$ is the resolution function that describes the distribution of $q$ values probed for the scattering vector setting, $\langle q\rangle$. The parameters entering the resolution function are estimated from the width of the direct beam at the detector. Equation (1) is used to fit the experimental data using a nonlinear least-squares procedure ${ }^{29}$.

FRET. Samples were purified on HPLC (Agilent Technologies 1200 series) using a BioSep-2000 column (Phenomenex) with an elution buffer containing $20 \mathrm{mM}$ Tris- $\mathrm{HCl}, \mathrm{pH} 8.0,100 \mathrm{mM} \mathrm{KCl}$ and $10 \mathrm{mM} \mathrm{MgCl}_{2}$. Ensemble FRET experiments were carried out in a JY-Horiba Fluoromax-3 spectrofluorimeter using a $60-\mu \mathrm{l}$ quartz cuvette (Hellma UK). Aliquots of $0.2 \mu \mathrm{l}$ of $200 \mu \mathrm{M}$ key or an unrelated oligonucleotide were directly added to the box sample and mixed by pipetting. Fluorescence spectra for each sample were recorded at $25^{\circ} \mathrm{C}$ with both Cy3 excitation at $530 \mathrm{~nm}$ and Cy5 excitation at $600 \mathrm{~nm}$, and corrected for background fluorescence and instrument response. The kinetic studies were performed with a $10-\mathrm{nm}$ slit centred at $665 \mathrm{~nm}$, and recorded each $30 \mathrm{~s}$ with high shutter speeds to minimize bleaching. The FRET efficiency was estimated by the RatioA method ${ }^{30}$.

26. Sander, B., Golas, M. M. \& Stark, H. Automatic CTF correction for single particles based upon multivariate statistical analysis of individual power spectra. J. Struct. Biol. 142, 392-401 (2003).

27. Sander, B., Golas, M. M. \& Stark, H. Corrim-based alignment for improved speed in single-particle image processing. J. Struct. Biol. 143, 219-228 (2003).

28. Pedersen, J. S., Posselt, D. \& Mortensen, K. Analytical treatment of the resolution function for small-angle scattering. J. Appl. Crystallogr. 23, 321-333 (1990).

29. Pedersen, J. S. Analysis of small-angle scattering data from colloids and polymer solutions: modeling and least-squares fitting. Adv. Colloid Interface Sci. 70, 171-210 (1997).

30. Clegg, R. M. et al. Fluorescence resonance energy transfer analysis of the structure of the four-way DNA junction. Methods Enzymol. 211, 353-388 (1992). 